UVX 2010 (2011) 49-55

DOI: $10.1051 / \mathrm{uvx} / 2011007$

(C) Owned by the authors, published by EDP Sciences, 2011

\title{
ThomX: A high flux compact $X$ ray source
}

\author{
C. Bruni ${ }^{1}$, N. Artemiev ${ }^{3}$, R. Roux ${ }^{1}$, A. Variola ${ }^{1}$, F. Zomer $^{1}$ and A. Loulergue ${ }^{2}$ \\ ${ }^{1}$ LAL CNRS, Université Paris-Sud 11, Bâtiment 200, 91898 Orsay, France \\ 2 Synchrotron SOLEIL, Saint-Aubin, France \\ ${ }^{3}$ ILE, École Polytechnique, CNRS, Palaiseau, France
}

\begin{abstract}
In the field of application of X-rays, there is a strong need for high brightness, tunable beam. The synchrotron radiation sources meet this demand, but the access time is limited, and they are large and expensive facilities. In this framework, we develop a compact monochromatic high flux X-ray source. The principle lies in obtaining X-ray by Compton backscattering of photons by relativistic electrons. This facility is relatively compact (about $70 \mathrm{~m}^{2}$ ) to be installed directly in a scientific laboratory, a hospital or an art museum. The backscattered photons are in the range of multi-keV energy, determined by the energy of the electrons and photons. Since there is a correlation between emission angle and energy of scattered photons, the spectral bandwidth of scattered photons can be controlled by simple collimation. The paper presents the ThomX X-ray source consisting of a $50 \mathrm{MeV}$ small storage ring and a laser coupled to a high gain Fabry Perot cavity.
\end{abstract}

\section{INTRODUCTION}

Classical bremsstrahlung X-ray tubes can generate typical X-ray radiation with a large spectrum and different intensities (rotating anode or microfocus tubes with adaptive optics). Compton X-ray scattering sources offer the possibility of compactness with respect to synchrotron radiation facilities, with more attractive characteristics (flux, directivity, monochromaticity, tunability) than X-ray tubes. The characteristics of the ThomX Compton source as designed in the Conceptual Design Report [1] are summarized in Table 1. With such parameters, the thomX source has the great advantage that the emitted photons energy cut-off allows one to provide hard X-ray beams, with a compact installation.

Currently the most ambitious projects, based on Compton back scattering interaction, aim to produce $10^{12}-10^{13} \mathrm{ph} / \mathrm{sec}$. The spectrum energy cut ranges in the few tens of $\mathrm{keV}$ with a bandwidth of the order of $1-10 \%$. Combining a low frequency linac with a small ring is a way to obtain a high repetition rate for the interaction (given by the ring circonference). It is the most adopted scheme for all the future sources which aim to produce a high average flux [2]. Another way to produce directly from the linac high repetition rate electron bunches, is to combine a DC gun to a superconducting linac. This scheme, which has been also studied for ThomX in the CDR, is used in the MIT project [3]. The LUCX [4] Japanese project is based on a multi-bunch rf electron gun to reach $1.310^{11} \mathrm{ph} / \mathrm{s}$ in $1 \%$ bandwidth. A key point is to obtain also a high repetition rate, high power laser, as in the ThomX and LUCX cases, with the development of Fabry perot cavity.

One of the most promising applications is the cultural heritage preservation and associated domains. Tunable and monochromatic hard X-rays can be obtained in Compton machines with the use of diaphragms (5-10\% bandwidth) or monochromators ( $0.1 \%$ bandwidth). Diffraction techniques, laminography and painting components chemical analysis should take advantages of such radiation sources. A great advantage should be acquired by exploiting complementarily the analysis worked out by employing ion sources like elastic scattering (RBS, ERDA), the use of nuclear reactions for the detection

This is an Open Access article distributed under the terms of the Creative Commons Attribution-Noncommercial License 3.0, which permits unrestricted use, distribution, and reproduction in any noncommercial medium, provided the original work is properly cited. 
Table 1. ThomX parameters range.

\begin{tabular}{|l|l|l|l|}
\hline \multicolumn{2}{|c|}{ Source explored range } & \multicolumn{2}{c|}{ Accelerator and laser } \\
\hline X ray energy & $50-90 \mathrm{keV}$ & Ring and injector energies & $50 \mathrm{MeV}$ \\
\hline Flux & $10^{11}-10^{13} \mathrm{ph} / \mathrm{s}$ & Charge & $1 \mathrm{nC}$ \\
\hline Bandwidth & $10 \%$ & Emittance (normalized rms) & $<5 \mathrm{~mm}$ \\
\hline Divergence & $<2 \mathrm{mrad}$ & $\begin{array}{l}\text { Transverses rms size of the electron } \\
\text { beam (at interaction point) }\end{array}$ & $70 \mu \mathrm{m}$ \\
\hline Repetition rate & $20-40 \mathrm{MHz}$ & Laser waist (at interaction point) & $80 \mu \mathrm{m}$ \\
\hline & & Intracavity average power & $>100 \mathrm{~kW}$ \\
\hline & & X-ray Repetition rate & $20-40 \mathrm{MHz}$ \\
\hline & & Laser waist (at interaction point) & $80 \mu \mathrm{m}$ \\
\hline
\end{tabular}

of light elements, and the hard X-ray techniques. By combining ion and photon measurement techniques on the same object, a more complete material analysis will be worked out. Non destructive analysis of paintings allows also underlying drawings to be revealed. The possibility to integrate such a source into a museum's laboratory gives to experts direct access to the masterpieces right in the Museum [5] and will avoid extremely important insurance, security and transportation costs, and troubles.

As far as the medical science applications are concerned an important benefit of such sources is given in imaging, by the development of the phase contrast method. Other applications are possible, for example, in static and dynamic imaging [6,7], 3D compressionless mammography [8], broncography, catheterless coronary arteries angiography [9] and in the K-edge radiography and therapy. An important feature of Compton sources is the tunability to specific X-ray energy obtainable by varying the energy of the electron beam (quadratic dependence) or the wavelength of the impinging laser (linear dependence). This is attractive since a resonant reaction can be triggered by the interaction between the $\mathrm{X}$ rays with a determined energy, and an electronic shell of a contrast agent (like K-shell extraction with a subsequent energy release by Auger cascade). Contrast agents (like Gadolinium or Platinum) based cancer imaging and therapy [6] could represent the real breakthrough of the Compton machines in various medical applications.

Other interesting domains can be identified in the fields that usually operate synchrotron radiation sources but that do not need a very high average flux. A spin-off of the SLAC laboratory has created a commercial Compton source for X-ray diffraction protein crystallography [10]. This is the first example of a working mini-synchrotron based on the Compton effect.

Moreover, due to the quadratic dependence of the scattered photons energy cut-off on the electron beam energy, it is easy to imagine harder photon production. This allows one to envisage different applications in the nuclear waste management and treatment industry [11] and in the field of nuclear isotope detection applied to infrastructures security. In this framework the atomic number identification by means of hard-X or gamma rays allows for nuclear application of the Compton scattering [12]. The high penetration power of these photons is extremely attractive when applied to security and to the radiography of shielded materials. In addition, the resonant detection can be applied; and one may take advantage from the relative monochromaticity degree of the photons pulses (scattered radiation).

\section{ACCELERATOR-LASER SYSTEM}

Figure 1 illustrates the view of the ThomX source. To fulfill the specifications of the ThomX accelerator, the linac must be carefully designed because most of the performances of the electron beam at the interaction point rely on the beam quality at the electron source, and mainly on the photo-injector. The linac will deliver one electron bunch each $20-50 \mathrm{~ms}$. We chose a photo-injector as electron gun, rather than the more classical thermoionic electron gun, for two main reasons. First, the photo-injector routinely demonstrates its capacity to produce a very low emittance electron beam. Second, the use of 


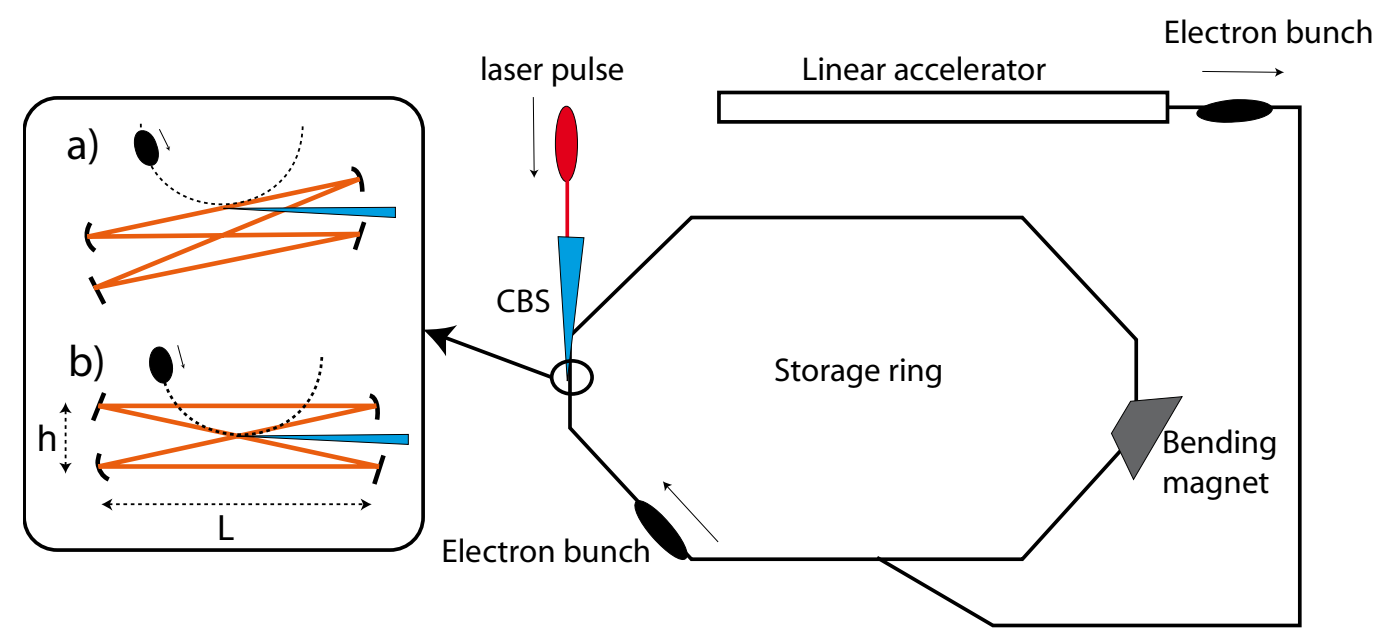

Figure 1. Schematic diagram of ThomX source. a) 2D bowtie planar cavity, b) 2D crossed planar cavity.

a thermionic gun would lead to a more complex and longer accelerator because it needs a prebunching cavity and a buncher. So our insight is that the photo-injector stands for the best technical choice. For this project reliability and cost are criteria as important as the performances of the electron beam. Hence, we will use a RF gun very similar to those built by LAL for the CLIC Test Facility 3 (CTF3) at CERN [13, 14].

Then the electron bunch is accelerated up to $50 \mathrm{MeV}$ in a $3 \mathrm{GHz}$ linear accelerator [15]. Via a transfer line, the electron bunch is stored in a ring. The injected bunch has to be on axis (no accumulation) and is very sensitive to every kind of mismatch in terms of positions and sizes. Mismatch would lead to longitudinal oscillations and emittance growth inducing X-ray flux reduction. The second difficulty rising at low energy is the high sensitivity to the wakefields. A careful design of the vacuum chamber has to be worked out and the impact of wakefields including Coherent Synchrotron radiation and Longitudinal Space Charge has to be investigated as well as a careful HOM care. The interaction takes place in a short straight section between two short dipoles. Due to the electron beam dynamics [16], the electron beam is stored in the ring only during $20-50 \mathrm{~ms}$, which is given by the bunch spacing from the linac. Mainly due to the stochastic effect of the Compton interaction and intrabeam scattering, the electron beam characteristics such as the emittance (related to the transverse dimension) and the energy spread (related to the bunch longitudinal dimension) are deteriorating with time. Beyond $50 \mathrm{~ms}$, the electron beam, which has suffered too much damage, is ejected. In fact, this electron bunch degradation reduces drastically the X-ray flux and the brilliance of the scattered photon source. During these 20-50 ms, the synchrotron damping time can be neglected, involving that the interaction takes place with the electron beam characteristic from the linear accelerator, explaining the importance of the electron gun quality at the exit of the linac.

To obtain high energy laser pulse in the Infrared frequency domain, a fiber laser, amplified in a rod type fiber [17] is coupled to a four mirrors high finesse Fabry-Perot cavity. We expect $100 \mathrm{~kW}$ average power in the cavity, with an initial laser power of $50 \mathrm{~W}$ and a cavity enhancement of 4000 . The four mirrors resonator is mechanically stable, especially when small laser waist is foreseen. In the case where the ratio between the height of the cavity and its length becomes small, a planar 2D cavity can be chosen instead of a 3D cavity [18]. Moreover, to further reduce the horizontal aperture of the vacuum chamber in order to conserve a small interaction angle, we chose the $2 \mathrm{D}$ crossed geometry (see fig. 1b) which shows the same optical properties as the 2D bowtie geometry (see fig. 1a). A planar optical cavity located in between the adjacent dipoles naturally fits the dipole bore gap. In addition, the optical cavity 


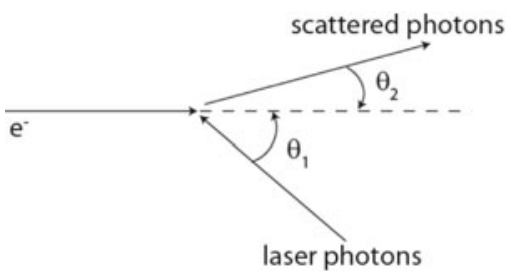

Figure 2. Scheme of Compton backscattering interaction.

mirrors can be accessed more easily being located out of the ring and scattered photons extraction cone is close to interaction point (thanks to the dipole curvature). The cavity round trip frequency is two times higher than the revolution frequency of the bunch in the ring. This leaves a possibility of storing two bunches in the ring to increase the X-ray flux.

\section{COMPTON SOURCE CARACTERISTICS}

The Compton back-scattering [19] characteristics are particularly attractive in the relativistic case. The energy spectrum of the scattered photons is characterized by an energy cut off $E_{x, \max }$ (see fig. 3a), that is the maximum energy gain of the photons with a quadratic dependence on the electron energy given by the Lorentz factor $\gamma$ (see fig. 3a) as : $E_{x, \max }=4 \gamma^{2} E_{l}$, with $\mathrm{E}_{l}$, the laser photon energy. This enables to produce high energy $\mathrm{X}$ rays with a low energy electron beam (around $50 \mathrm{MeV}$ ), leading to a compact installation.

The scattered photons gain the maximal energy (i.e the energy cut off) in the so-called "head-on" collision. For non zero collision angles $\theta_{1}$ (see fig. 2) the maximum energy is reduced. Another important characteristic for ThomX, is univocal relation between the scattered photon energy $\mathrm{E}_{x}$ and its scattering angle $\theta_{2}$ as expressed by the following relation [20]:

$$
E_{x}=\frac{E_{l}\left(1-\beta \cos \left(\pi-\theta_{1}\right)\right)}{1-\beta \cos \theta_{2}+E_{l} / E_{e}\left(1-\cos \left(\theta_{2}+\theta_{1}-\pi\right)\right)}
$$

With $\mathrm{E}_{e}$ the electron energy and $\beta$ the velocity of the electrons normalized to the speed of light. This is very effective for selecting the spectral bandwidth, and consequently to obtain a monochromatic beam, by setting up a simple diaphragm. At relativistic energies, the emission cone is extremely narrow



a)

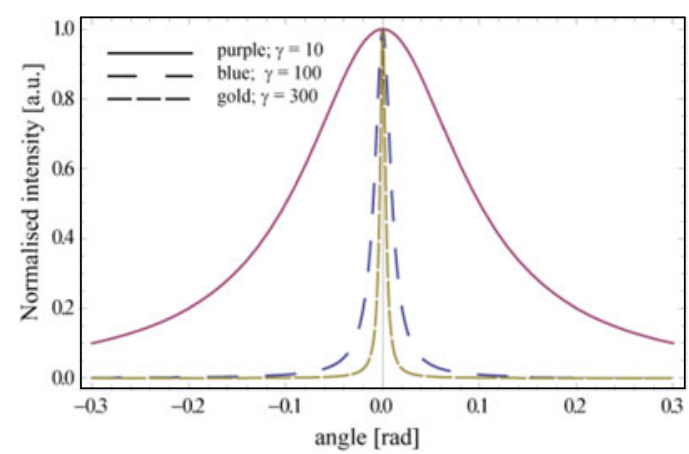

b)

Figure 3. a) Differential cross section as a function of the scattered photon energy. The assumed initial photon energy is $2.4 \mathrm{eV}$ and electrons energy is $50 \mathrm{MeV}$. b) Angular distribution of the backscattered photons for three different electron energies. 


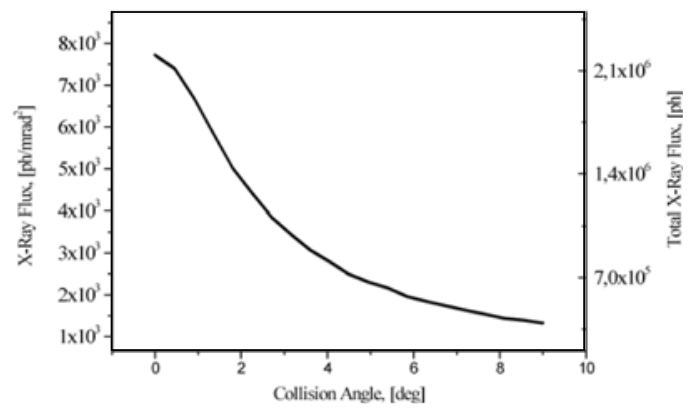

a)

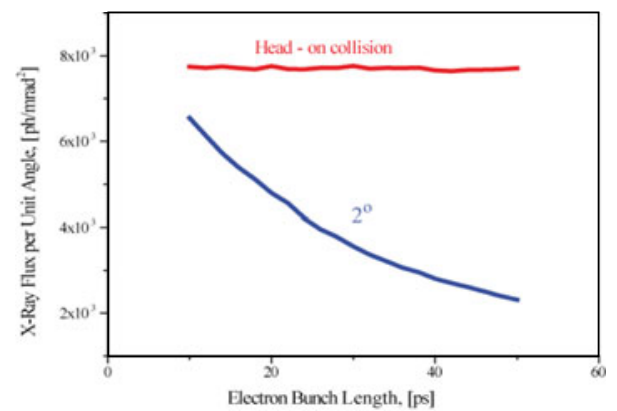

b)

Figure 4. a) Number of photons per unit angle (left axis) and number of photons defined as Flux/ $\mathrm{f}_{\text {rep }}$ (right axis) per collision versus the angle of interaction between the laser and the electron beams. b) number of photons per unit angle per collision versus r.m.s. electron bunch length. This is calculated for 2 degrees angle of collision (blue line) and for the head-on (red line) configurations. Monte Carlo simulations have been performed with a home made code.*

(see fig. 3b), thus allowing a photon beam brilliance increase. The limit of the angular shrinking is given, in a real experiment, by the fact that the Compton flux is not generated by a single particle but rather by an electron beam with a natural divergence at the interaction point. The resulting angular aperture of the emitted photons will then be given by the convolution between the Compton $1 / \gamma$ cone and the electron beam divergence. This is extremely important since, taking into account the angle-energy dependence for the scattered photons, a too strongly divergent electron beam will affect not only the brilliance, but also the possibility to select a monochromatic beam by using diaphragms.

Hereafter we assume a single Compton collision of a relativistic electron bunch with a high power laser pulse. The luminosity of the interaction [21] is given by:

$$
L=N_{e} N_{l} \mathrm{f}_{\text {rep }} \frac{\cos \phi}{2 \pi} \frac{1}{\sqrt{\sigma_{y e}^{2}+\sigma_{y l}^{2}} \sqrt{\left(\sigma_{x l}^{2}+\sigma_{x e}^{2}\right) \cos ^{2} \phi+\left(\sigma_{z e}^{2}+\sigma_{z l}^{2}\right) \sin ^{2} \phi}}
$$

where $\mathrm{N}_{\mathrm{e}}, \mathrm{N}_{\gamma}, \mathrm{f}_{\text {rep }}, \phi, \sigma_{x, y, z, e}, \sigma_{x, y, z, l}$ indicate respectively the number of electrons and photons per bunch, the repetition frequency, the half of the angle of collision $\theta_{1}$ and the three dimensional bunches r.m.s. sizes for the electron (index e) bunch and photon (index 1) pulse. The total flux of the scattered photons is linked to the luminosity via the Compton cross section as: Flux $=\sigma_{\text {Compton }}$ L. To maximize the flux, the repetition rate has to be high, $20 \mathrm{MHz}$ in our case. Then the bunch charge and the photons pulse energy should be high, with a limitation depending principally on the laser and accelerator systems used. The bunch length becomes even more important in the case of some interaction angle is introduced. Figure 4a shows the evolution of the X-ray flux with the collision angle. A reduction of the flux by a factor two is obtained between a head on collision and a $2^{\circ}$ collision. Due to the insertion of the optical cavity, and its stability, the interaction angle is set at $2^{\circ}$ in the ThomX case. For such collision angle, the electron bunch should not undergo lengthening to avoid losing too flux (see fig. 4b).

We have also to care about the brilliance, which takes into account the energy/scattering angle dependence. Figure 5 highlights that the maximum brilliance does not correspond to the maximum flux. There is a trade off between beam size and beam divergence. The emittance (see Table 1) of an electron beam can be viewed as the product of its transverse dimension by its divergence. If the focusing is stronger, the divergence is increased, and then the spectrum is larger. As a consequence the total flux increases, but the brilliance, which takes into the account the number of photons per unit angle, per unit source area, and per $0.1 \%$ of energy bandwidth, has a maximum around $65 \mu \mathrm{m}$ in ThomX case. This point is a major interest for the X-ray source since the brilliance is the key parameter for such important 


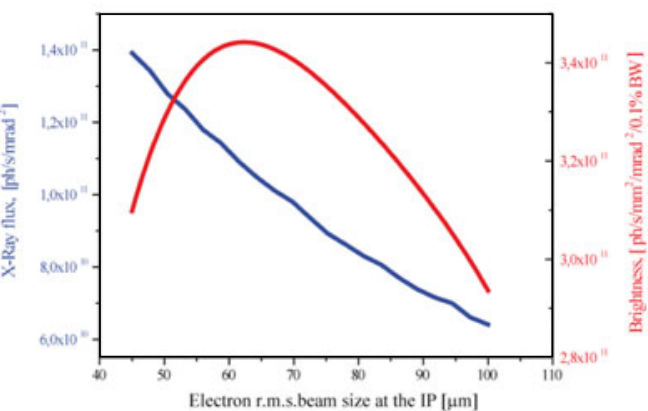

a)



b)

Figure 5. a) X-ray flux per unit angle (blue curve) and brightness (red curve) versus the r.m.s. electron beam waist size. The X-ray flux per unit angle should be divided by repetition rate to obtain the number of photons per unit angle quoted in figure 4 . Figure 4 highlights the relation between the number of photons and the number of photons per units angle. If the number of photons is multiplied by the repetition frequency, one can obtain the total flux quoted in table 1 . The assumed collision angle is 2 degrees and the collision repetition frequency is $20 \mathrm{MHz}$. b) Number of photons per unit angle versus electron bunch time jitter with respect to laser pulse calculated for 2 degree collision angle (blue curve) and the head-on collision (red curve). Monte Carlo simulations have been performed with a home made code.*

X-ray applications as imaging, tomography. These values are given for the first pass interaction. Then, due to the interaction itself and collective effects of the electron beam, the flux decreases. When the flux dropped by a factor two after $20-50 \mathrm{~ms}$, a new bunch is stored to reach again the maximum flux.

When crossed collisions are taken into account, the possible time mismatching plays a crucial role in scattering efficiency. The number of scattered photons per unit angle drops drastically with the increase in collision angle when the electron bunch and the laser pulse interact with non-zero time delay. However, for the 2 degrees collision angle and given durations of the pulses the tolerance on the time jitter between the two pulses looks reasonable within $15 \mathrm{ps}$.

\section{CONCLUSION}

A compact hard X-ray source has been designed. Although the brilliance is smaller than the third generation synchrotron light source, the energy range of the scattered photons competes with hard X-ray sources with a rather more compact installation. Its flux is sufficient for the most of scientific application. The properties of the scattered photons enable to obtain monochromatic beams. The ThomX conceptual design report is already available [1], and the technical design report will be available during 2011.

\section{Acknowledgments}

The authors acknowledge the French partners of the project: R. Chiche, J. Haissinski, M. Jacquet, M. Lacroix, Y. Peinaud, R. Cizeron, A. Gonnin, R. Marie, J. Bonis, M. Joré, D. Jehanno, L. Meignien, B. Mercier, B. Mouton, C. Prevost, V. Soskov, M. Tran, T. Vandenberghe, G. Wormser, LAL, C. Benabderrahmane, P. Brunelle, L. Cassinari, J.C. Denard, M. Diop, J.M. Filhol, N. Guillotin, N. Hubert, M. Labat, J.P. Lavieiville, P. Lebasque, P. Marchand, JL Marlats, F. Marteau, R. Nagaoka, F. Ribeiro, SYNCHROTRON SOLEIL, P.Balcou, E. Cormier, M.C. Nadeau, CELIA (Université de Bordeaux 1), P. Walter, C2RMF (Ministère de la Culture), R. Flaminio, C. Michel, L. Pinard,

*The main results have been validated by the comparison with the general analytical formula for the Compton collision luminosity of two Gaussian bunches crossing, where Gaussian distributions are assumed and the hourglass effect is neglected (assuming constant beams sizes during the collision, so without taking into account the cross effect of the laser Rayleigh length, the IP beta function and their correlation with the bunches lengths). 
B. Sassolas, LMA ( VILLEURBANNE), J.P. Brasile, M.C. Nadeau, THALES, G. Le Duc, A. Bravin, from ESRF (Grenoble).

\section{References}

[1] ThomX conceptual design report available at http://hal.in2p3.fr/in2p3-00448278_v1/

[2] Z. Huang and R. Ruth, Phys. Rev. Lett. 80, 976 (1998), E. Bulyak et al., Nucl.Inst. and Meth. A 487 (2002) 241-248

[3] W.S. Graves, W. Brown, F.X. Kaertner, D.E. Moncton, Nucl. Inst and Meth. A 608, S103 (2009),

[4] J. Urakawa, In press in Nuclear Instrum. And methods A (2010)

[5] See the Louvre laboratory web site: http://www.c2rmf.fr/

[6] M. Ando and C. Uyama, "Medical Application of Synchrotron Radiation", Springer and Verlag (1998); P. Suortti, W. Thomlinson, Phys. Med. Biol. 48 (2003) R1; G. Margaritondo et al., Rev. Nuov. Cim. 7 (2004) 1;

[7] E. Rubistein et al. Proc SPIE 314 (1981) 42

[8] F. Carroll, Journal of Cellular Biochemistry 90 (2003) 502.

[9] E.G. Bessonov et al arXiv:physics/0405003 v1 (2004).

[10] http://www.lynceantech.com/

[11] R. Hajima et al., Journal of Nuclear Science and Technology, 45 (2008) 441

[12] C.P.J. Barthy and F.V Hartemann. UCRL-TR 206825, LLNL laboratory report

[13] C. Travier et al., Nucl. Instr. Meth. in Phys. Res. A 304 (1991) 285

[14] J. Brossard, M. Desmons, B. Mercier, C. Prevost, R. Roux, proceedings of EPAC, 828 (2006) http://accelconf.web.cern.ch/accelconf/

[15] R. Belbeoch et al., Rapport d'études sur le projet des linacs injecteurs de LEP (LIL), LAL/RT 82-01 (1982)

[16] A. Loulergue, C. Bruni, J. Haissinski, A. Variola, M. Lacroix, M. Jore, T. Vandenberghe, proceedings of IPAC, 4650 (2010) http://accelconf.web.cern.ch/accelconf/

[17] Y. Zaouter, D. N. Papadopoulos, M. Hanna, J. Boullet, L. Huang, C. Aguergaray, F. Druon, E. Mottay, P. Georges, E. Cormier, Optics letters 33 (2008) 107

[18] F. Zomer, Y. Fedala, N. Pavloff, V. Soskov, A. Variola, Appl. Opt. 48, 6651 (2009)

[19] J.D. Jackson; Classical Electrodynamics, Third Edition, 1999. John Wiley and Sons

[20] R. J. Loewen, A compact light source: design and technical feasibility study of a laser-electron storage ring X-ray source, SLAC-Report-632 (2003).

[21] T. Suzuki, "General formulae of luminosity for various types of colliding beam machines" KEK 76-3 July 1976 